\title{
Failure of a recombinant Schistosoma bovis-derived glutathione S-transferase to protect cattle against experimental Fasciola hepatica infection
}

\author{
J. De Bont ${ }^{\mathrm{a}}$, E. Claerebout ${ }^{\mathrm{b}}$, G. Riveau ${ }^{\mathrm{a}}$, A.M. Schacht ${ }^{\mathrm{a}}$, \\ K. Smets ${ }^{\text {b }}$, G. Conder ${ }^{\text {c }}$, D.A. Brake ${ }^{\text {c }}$, A. Capron ${ }^{\text {a }}$, J. Vercruysse ${ }^{\text {b,* }}$ \\ a Inserm U547, Institut Pasteur de Lille, Rue du Prof Calmette, Lille 59019, France \\ ${ }^{\mathrm{b}}$ Laboratory of Parasitology, Faculty of Veterinary Medicine, Ghent University, \\ Salisburylaan 133, 9820 Merelbeke, Belgium \\ c Veterinary Medicine Research and Development, Pfizer Animal Health Group, Pfizer Inc., \\ Eastern Point Road, Groton, CT 06340, USA
}

Received 9 August 2002; received in revised form 9 December 2002; accepted 9 December 2002

\begin{abstract}
The potential of a recombinant Schistosoma bovis 28-kDa glutathione S-transferase (rSb28GST) to protect cattle against Fasciola hepatica was tested in a vaccination trial. Thirty two calves were randomly divided into four groups of eight animals. Calves of the three vaccine groups received two intramuscular injections at 3 weeks interval, of $0.250 \mathrm{mg} \mathrm{rSb} 28 \mathrm{GST}$ in either aluminium hydroxide $\left(\mathrm{Al}(\mathrm{OH})_{3}\right)$, Quil A, or PBS emulsified in an equal volume of Freund's complete adjuvant (FCA).

Animals of the control group received injections of $\mathrm{Al}(\mathrm{OH})_{3} / \mathrm{PBS}$ only. All animals were challenged orally with a total of 360 metacercariae of $F$. hepatica, spread over 6 weeks.

All groups of vaccinated animals produced measurable IgG antibody titers to rSb28GST after vaccination. Animals immunised with FCA adjuvanted vaccine had the highest and more durable antibody titers and only sera from this group recognised an approximately $24 \mathrm{kDa}$ protein band from $F$. hepatica, that is thought to be a $F$. hepatica GST. Despite a good antibody response differences in cumulative faecal egg output between the groups were not statistically significant. In addition, no significant difference was found between groups in terms of total worm numbers or percentage of immature flukes recovered at necropsy. In conclusion, the recombinant $S$. bovis $28 \mathrm{kDa}$ GST was not found to adequately protect cattle against experimental $F$. hepatica challenge, using either aluminium hydroxide, Quil A or FCA as adjuvant.
\end{abstract}

(C) 2003 Elsevier Science B.V. All rights reserved.

Keywords: Fasciola hepatica; Cattle-trematoda; Vaccine; Glutathione S-transferase

\footnotetext{
* Corresponding author. Tel.: +32-9-264-7390; fax: +32-9-264-7496.

E-mail address: jozef.vercruysse@rug.ac.be (J. Vercruysse).
} 


\section{Introduction}

Infection of ruminants with Fasciola hepatica and Fasciola gigantica causes significant economic loss estimated at over US\$2000 million per annum to the agricultural sector worldwide with over 600 million animals infected (Spithill et al., 1999a). Cattle acquire resistance to challenge infection with $F$. hepatica or $F$. gigantica when previously sensitized with a primary homologous infection (Mulcahy et al., 1999, Spithill et al., 1999b), although this resistance may not be totally immunologically based (Mulcahy et al., 1999). There have been many attempts to vaccinate cattle with various liver fluke extracts, such as crude somatic antigens and excretory/secretory antigens, but also with irradiated attenuated vaccines and various defined antigens (reviewed by Spithill et al., 1999a). The mean level of reduction in worm burdens observed in cattle with different antigens was in the range of $43-72 \%$, suggesting that the control of fasciolosis by immunological intervention may be an achievable goal (Spithill et al., 1999a).

Heterologous immunity between Schistosoma and Fasciola spp. has also been demonstrated in cattle, with reductions in liver fluke worm recoveries of 30-84\% in calves which had been exposed 8-10 weeks earlier to cercariae of S. bovis (Sirag et al., 1981; Yagi et al., 1986). Among the various antigens currently tested against human schistosomiasis, the recombinant $S$. haematobium glutathione S-transferase (Sh28GST) is clearly the most advanced vaccine candidate. It has now reached phase 2 clinical trials in man (Capron et al., 2002). During pre-clinical studies of this schistosome vaccine, the potential of a recombinant $S$. bovis-derived GST (Sb28GST) to protect cattle against $S$. mattheei infection was tested in Zambia (De Bont et al., 1997; Grzych et al., 1998). These animals were challenged naturally during 9 months on a farm known to be endemic for both S. mattheei and $F$. gigantica. At necropsy, a striking difference was observed between vaccinated and control groups in the severity of $F$. gigantica infections (unpublished data). Most immunized animals remained uninfected, whereas liver flukes were found in all controls, sometimes with severe lesions. In both groups, individual variations in the severity of Fasciola infections were found directly proportional with the intensity of the $S$. mattheei infection (De Bont, 1995). Unfortunately, no quantitative evaluation of the level of protection was carried out. Therefore, the present study was carried out to test the potential of the rSb28GST to protect cattle against $F$. hepatica. Because protection against $F$. hepatica varies dramatically with the adjuvant used (Spithill et al., 1999a), the rSb28GST antigen was combined with freund complete adjuvant (FCA), Quil A or aluminium hydroxide $\left(\mathrm{Al}(\mathrm{OH})_{3}\right)$ in different groups of animals.

\section{Materials and methods}

\subsection{Animals}

A total of 32 helminth naive Holstein female calves, 8-10-month old, were purchased. Authorization to use these calves for the present study was obtained from the Ethical Committee of the Faculty of Veterinary Medicine, Ghent University. Blood and faecal samples were examined to confirm that animals were worm free. They were stabled individually at 
the experimental farm of Ghent University for the duration of the trial. The calves were fed corn silage ad libitum and $1 \mathrm{~kg}$ per day of concentrate and had free access to drinking water. The animals were observed daily for clinical signs of fasciolosis.

\subsection{Immunization and infection schedule}

The same lot of recombinant Sb28GST as used in Zambia was provided by the Institut Pasteur at Lille, France. It was prepared as previously described (Trottein et al., 1992; Boulanger et al., 1994).

The trial was conducted as a randomised, blinded, controlled study. The 32 animals were randomly divided into four groups of 8 animals each. They all received two intramuscular injections at 3 weeks interval, the first one (10 April 2000, day 0) in the left neck, the second one (2 May 2000, day 22) in the right neck. Immunogens were randomly assigned to the treatment groups. Animals of the adjuvant control group received injections of $\mathrm{Al}(\mathrm{OH})_{3} / \mathrm{PBS}$ only. Animals of the vaccinated groups received injections of $0.250 \mathrm{mg}$ rSb28GST in either $\mathrm{Al}(\mathrm{OH})_{3}$, Quil A, or PBS emulsified in an equal volume of FCA. The day following each injection (day 1 and 23), the injection sites were carefully examined for the presence of reactions and a general clinical observation was carried out to detect any abnormal sign.

Starting from the fourth week after the second injection, all animals were challenged orally with metacercariae of $F$. hepatica (Baldwin Aquatics, Monmouth, OR): 20 metacercariae per day, 3 days per week during 6 weeks (total 360 metacercariae per animal, from day 49 to day 88). All animals were slaughtered for worm counts 10 weeks after the last challenge (days 154 and 155).

\subsection{Samplings}

Faecal samples were collected for egg counts 3 days per week starting from day 133 until day 151 (total of 9 samplings per animal). Blood was collected weekly during the whole trial for determination of parasitological, immunological and pathological parameters. The body weight was measured at the start of the study and then every 4 weeks, until the end of the study.

\subsection{Parasitological methods}

Faecal egg counts were determined according to a concentration-flotation method using zinc chloride and expressed as number of eggs per gram faeces (EPG). Cumulative faecal egg counts were calculated as described by Vercruysse et al. (1993). Worm recovery at necropsy was carried out as follows: the livers were recovered with intact gall bladder that was tied to prevent bile and fluke out-migration. After removing the gall bladder, flukes were "milked out" by pressing and working toward the open duct along known major bile duct branches. The bile ducts were opened with blunt-blunt scissors and the flukes removed with blunt forceps. Then, the entire liver was turned upside down in the tray and soaked with hot tap water (not scalding, but hotter that you can comfortably leave your hand in) for $1 \mathrm{~h}$, and the flukes were collected in the water. Finally, the liver was cut into $1 \mathrm{~cm}$ slices and placed in a 12-151 bucket filled to top with hot tap water. After allowing to stand and 
mixing to free the flukes from the slices, the supernate was poured through $200 \mu \mathrm{m}$ sieves to retrieve flukes. This procedure was repeated two times. Flukes from washes were collected and counted using a back-lighted magnifier.

Eggs were collected from the gall bladder, washed with tap water and incubated in water at $25^{\circ} \mathrm{C}$ for 5 weeks. A total of 100 randomly selected eggs per animal was examined to evaluate the level of maturation.

\subsection{Pathological parameters}

PCV and levels of haemoglobin were determined on day 140. Levels of SGOT, $\gamma$-GT and alcaline phosphatase were measured on day 140, 147 and 151. Levels of total bilirubin were measured on day 140 and 147. Finally, levels of total blood protein were measured on day 151. Prior to analysis of the data, arithmetic mean values of the different sampling days were calculated for each animal.

At necropsy, liver and bile ducts were examined for macroscopic lesions and the thickness of the wall of the main bile duct was measured.

\subsection{Immunological parameters}

Differential blood cell counts were determined on day 140.

The specific IgG and IgA serum antibody responses to the rSb28GST antigen were measured on days 0,22 (second injection), 49 (before infection), 56 (during infection period), 91 (after last infection), 133 (start of faecal egg excretion) and 151 (necropsy) using an ELISA, as described by De Bont et al. (1997) and expressed as geometric mean titres.

The serological IgG1 responses induced by vaccination against $F$. hepatica or S. bovis whole worm antigens were evaluated on days 0,49 and 151 by Western blotting using pooled sera from each group. Thirty five microgram of $F$. hepatica and $S$. bovis whole worm antigens were fractionated using 10\% SDS-PAGE under reducing conditions and then blot transferred onto a nitrocellulose membrane (Scheider and Schuell $-0.45 \mu \mathrm{m}$ ). The blot sections were blocked during $3 \mathrm{~h}$ in PBS $+0.5 \%$ gelatine (powder food grade, Merck). Then, the blot sections were incubated overnight with pooled sera (diluted 1/100 in PBS $0.5 \%$ gelatine) from the different groups. After washing, the conjugate (rabbit anti-bovine IgG1-HRPO, Sigma, 1/4000 in PBS 0.5\% gelatine) was added for $1 \mathrm{~h}$. Recognized antigens were visualised by adding 4-chloro-1-naphtol (Sigma-solution for Western blotting).

\subsection{Data analysis}

Data are presented as arithmetic group means $( \pm$ S.E.M.) or geometric means $(+$ range $)$. Indicators of worm fitness (cumulative faecal egg counts, total worm burden, percentage of mature worms and percentage of embryonated eggs) and pathological parameters associated with fasciolosis (anaemia, bilirubinaemia, increased liver enzyme levels and thickness of the bile duct) were expected to be lower in the vaccinated animals, while indicators of hosts' fitness (body weight, total blood protein level) and immune responses (serum antibody levels and eosinophil counts) were expected to be increased. The significance of 
differences between the four groups was assessed using a Kruskal-Wallis one-way analysis of variance, followed by a one-tailed Mann-Whitney $U$-test for pairwise comparison of each vaccinated group with the adjuvant controls. Probability $P<0.05$ were considered to indicate significant differences.

\section{Results}

\subsection{Parasitological data}

All animals were found positive on faecal egg examination at least once in the nine samplings, indicating that the method used for infection was successful. The egg excretion pattern was similar in all groups, with maximum geometric mean egg counts between 6 and 11 EPG (individual counts 0-36 EPG) in the last week before necropsy (Fig. 1). Differences in cumulative faecal egg counts between the four groups were not statistically significant $(P \leq 0.85)$ (Table 1).

Worm counts are presented in Table 1 . There was no significant difference between the groups in total worm numbers $(P \leq 0.88)$ or in the percentage of immature flukes recovered at necropsy $(P \leq 0.39)$.

In contrast, a difference was observed in the capacity of the eggs to mature after 5 weeks of incubation in vitro. A significantly higher percentage of non-embryonated eggs was

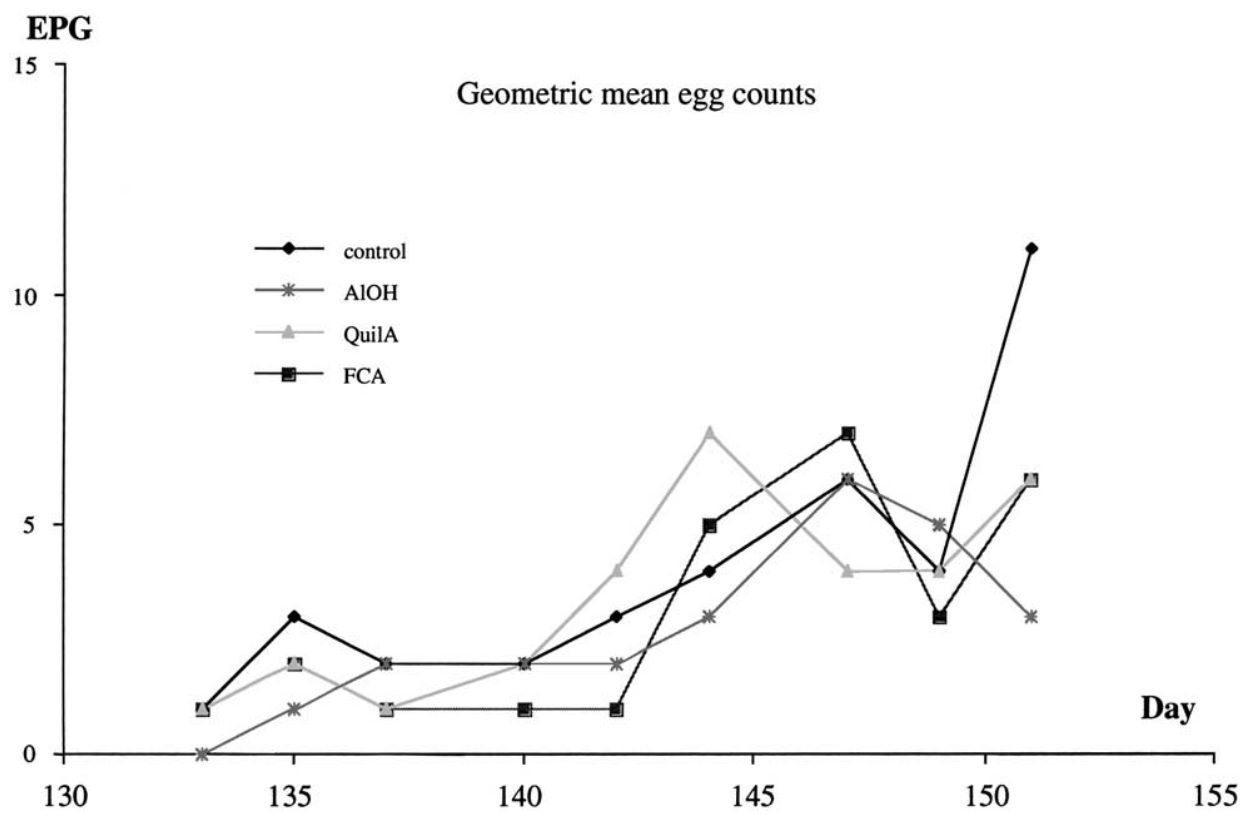

Fig. 1. Geometric mean egg counts from days 133 to 151 of calves vaccinated with rSb28GST and FCA, rSb28GST and Quil A, rSb28GST and $\mathrm{Al}(\mathrm{OH})_{3}$ or $\mathrm{Al}(\mathrm{OH})_{3}$ alone (controls). 
Table 1

Geometric mean cumulative faecal egg counts, non-embryonated eggs (\%), geometric mean total worm counts, immature flukes $(\%)$, arithmetric mean weight gains $(\mathrm{kg})$, arithmetric mean thickness of the bile ducts $(\mathrm{mm})$ and arithmetric mean serum $\gamma \mathrm{GT}$ levels (iU/l) of calves vaccinated with $\mathrm{Al}(\mathrm{OH})_{3}$ alone (controls) or vaccinated with rSb28GST formulated in $\mathrm{Al}(\mathrm{OH})_{3}$, Quil A, or FCA

\begin{tabular}{|c|c|c|c|c|c|c|c|c|}
\hline \multirow[t]{2}{*}{ Variables } & \multicolumn{2}{|c|}{ Controls } & \multicolumn{2}{|c|}{$\mathrm{Al}(\mathrm{OH})_{3}$} & \multicolumn{2}{|c|}{ Quil A } & \multicolumn{2}{|l|}{ FCA } \\
\hline & Mean & $\begin{array}{l}\text { S.D. or } \\
\text { range }\end{array}$ & Mean & $\begin{array}{l}\text { S.D. or } \\
\text { range }\end{array}$ & Mean & $\begin{array}{l}\text { S.D. or } \\
\text { range }\end{array}$ & Mean & $\begin{array}{l}\text { S.D. or } \\
\text { range }\end{array}$ \\
\hline EPG & 79 & $21-255$ & 56 & $15-182$ & 69 & $10-230$ & $55^{*}$ & $5-137$ \\
\hline Non-embryonated eggs (\%) & 6 & $1-13$ & 9 & $3-17$ & 15 & $0-40$ & 29 & $3-100$ \\
\hline Worm counts & 26 & $13-39$ & 25 & $14-45$ & 29 & $18-52$ & 29 & $16-42$ \\
\hline Immatures (\%) & 23 & $8-44$ & 21 & $0-33$ & 32 & $22-48$ & 26 & $14-44$ \\
\hline Weight gain & 77 & 37.9 & 69 & 11.4 & 68 & 11.7 & 72 & 11.5 \\
\hline Thickness bile duct & 2.59 & 0.91 & 2.97 & 1.28 & 2.25 & 0.49 & 3.05 & 0.47 \\
\hline$\gamma \mathrm{GT}$ & 128 & 64.6 & 83 & 47.1 & $59^{*}$ & 29.8 & $67^{*}$ & 29.0 \\
\hline
\end{tabular}

$* P<-0.05$.

recovered from the FCA vaccinated animals, compared to the control group $(P<0.01$, Table 1). On average, more eggs had also failed to embryonate in the other vaccinated groups, but these results were not significantly different from the control group.

\subsection{Clinical and physiopathological data}

Two animals died during the trial and were autopsied, but these events were considered to have no relationship to the investigational vaccine. The first animal $\left(\mathrm{Al}(\mathrm{OH})_{3}\right.$ adjuvant group on day 29) died following self-injury, the second animal (Quil A adjuvant group on day 103) died following a torsion of the mesentary. No adverse clinical reaction to the immunisations, either local or general, was observed during the trial.

The average body weight gains over the period of the trial (Table 1) were not significantly different between the groups $(P \leq 0.81)$.

Blood values tested at day 140, 147 and 151 were within normal ranges (data not shown), except for $\gamma$-GT. Mean $\gamma$-GT values exceeded the normal range of $0-31 \mathrm{iU} / 1$ (Blood and Radostits, 1989) in all groups, and were significantly higher in the control group than in the groups that were vaccinated with FCA $(P \leq 0.04)$ and QuilA $(P \leq 0.02)$ as adjuvants (Table 1).

The average thickness of the main bile duct wall in the four groups of animals was around 2-3 mm (Table 1), with no significant difference between the groups $(P \leq 0.20)$.

\subsection{Immunological data}

Moderate eosinophilia was present in all groups on day 140. Mean blood eosinophil numbers $\left(10^{9} / 1\right)$ were $8 \pm 6.6$ in the control group, $9 \pm 4.8$ in the $\mathrm{Al}(\mathrm{OH})_{3}$ adjuvant group, $7 \pm 7.8$ in the Quil A adjuvant group and $6 \pm 4.5$ in the FCA adjuvant group. No significant difference was observed between the groups $(P \leq 0.67)$.

The average IgG antibody responses to rSb28GST in the different groups of cattle on day 0,22 (second injection), 49 (before infection), 56 (during infection period), 91 (after 


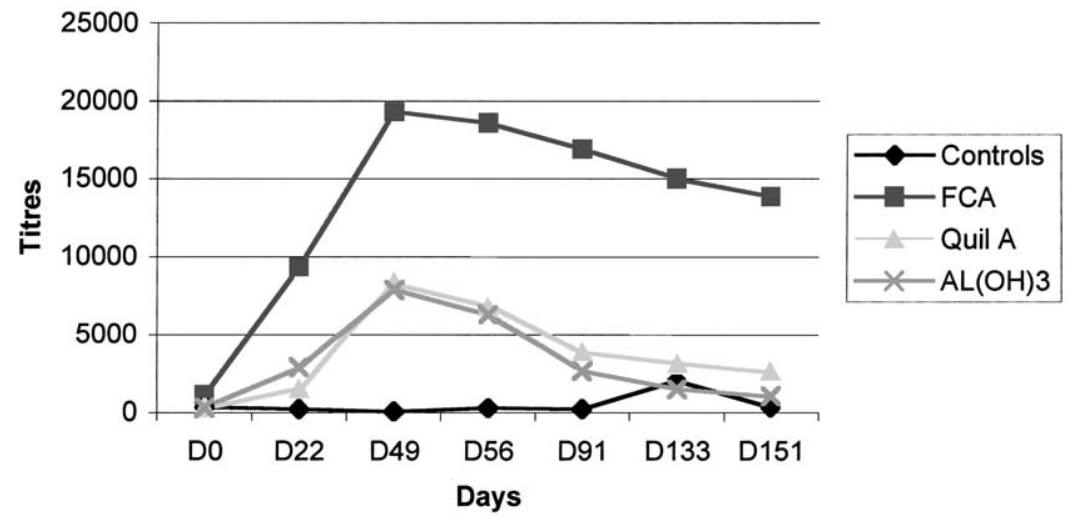

Fig. 2. Geometric mean IgG antibody titres to rSb28GST on days $0,22,49,56,91,133$ and 151 in calves vaccinated with rSb28GST and FCA, rSb28GST and Quil A, rSb28GST and $\mathrm{Al}(\mathrm{OH})_{3}$ or $\mathrm{Al}(\mathrm{OH})_{3}$ alone (controls).

last infection), 133 (start of faecal egg excretion) and 151 (necropsy) are shown in Fig. 2. Results indicate a robust IgG antibody response to the vaccine antigen at day 49 in the three vaccinated groups, when compared to the controls. The highest and most durable antibody response was obtained when using the water-in-oil adjuvant FCA for vaccine formulation. $\mathrm{IgG}$ titres decreased in all vaccinated groups during the infection period (from day 49

Fasciola hepatica

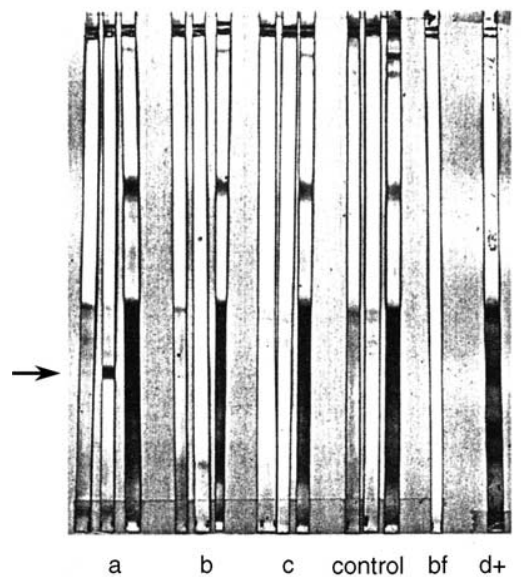

Swap S.bovis

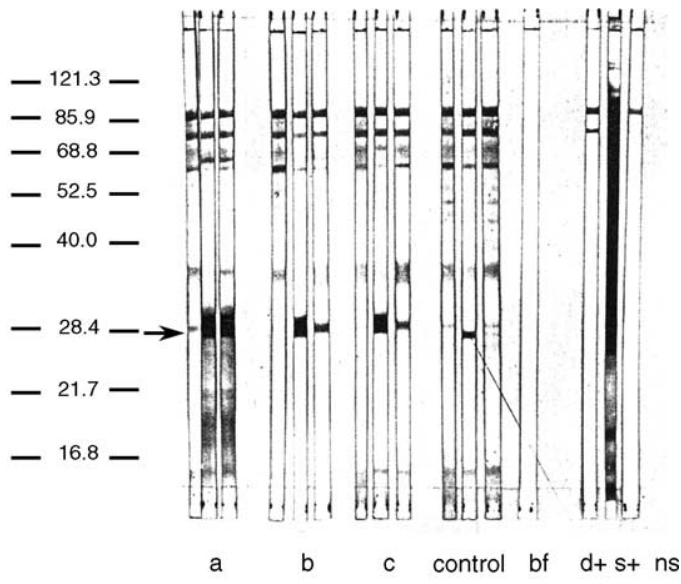

Fig. 3. Serum antibody responses to the vaccinations monitored by Western blotting against Fasciola hepatica (panel A) and Schistosoma bovis (panel B) whole worm extracts. Serum samples from individual animals were taken on days 0 (first lane), day 49 (second lane) and day 151 (third lane) and pooled for each group: (a) rSb28GST and FCA, (b) rSb28GST and Quil A, (c) rSb28GST and $\mathrm{Al}(\mathrm{OH})_{3}$, control: $\mathrm{Al}(\mathrm{OH})_{3}$ alone, bf: buffer, $\mathrm{d}+$ : serum from rabbit immunised against $F$. hepatica, s+ serum from rabbit immunised against $S$. bovis, ns: serum from non-immunised rabbit. 
onwards). In contrast to IgG, no serum IgA titers against rSb28GST were detected during the experiment (data not shown).

Following two dose vaccination (day 49), pooled sera from each of the immunised groups recognised a $28 \mathrm{kDa} S$. bovis protein which was not recognised by the pooled sera from the control group (Fig. 3B). After infection with $F$. hepatica (day 151), the intensity of staining of the $28 \mathrm{kDa}$ band by serum antibodies had fainted in the Quil $\mathrm{A}$ and $\mathrm{Al}(\mathrm{OH})_{3}$ adjuvant groups, but not in the FCA group. No additional $S$. bovis protein bands were recognised at the end of the vaccine trial, compared to day 49. Only sera from the FCA adjuvant group identified a $F$. hepatica $24 \mathrm{kDa}$ protein following vaccination (day 49) (Fig. 3A). Post-challenge antisera from all groups recognised additional $F$. hepatica antigens, however reactivity to the $24 \mathrm{kDa}$ antigen was consistently absent in groups immunized with either Quil A or $\mathrm{Al}(\mathrm{OH})_{3}$ vaccines.

\section{Discussion}

The ELISA results indicate a robust antigen-specific IgG antibody response immediately prior to challenge in the three vaccinated groups, when compared to non-immunised controls. Antisera recognised a $28 \mathrm{kDa}$ S. bovis antigen on Western blot, which is presumably the native $S$. bovis OST present in adult worm extracts Moreover, sera from calves that were immunised with FCA recognised an approximately $24 \mathrm{kDa}$ protein from $F$. hepatica. Based on its molecular weight, this protein is thought to be a homologous $F$. hepatica GST (Cervi et al., 1999). However, infection with $F$. hepatica did not boost the recognition of the $24 \mathrm{kDa} F$. hepatica antigen in the other vaccinated groups. Moreover, antibody titres decreased as soon as the experimental infections started, possibly due to the formation of complexes with worm antigens, which also suggests cross-reactivity with $F$. hepatica antigens. A similar decrease in specific IgG titres during $S$. mattheei challenge infections was observed in Zambia (De Bont et al., 1997). In contrast with the results of De Bont et al. (1997), an experimental challenge with $F$. hepatica was not capable to boost the production of IgA antibodies in a similar way as $S$. matthee $i$ and $F$. gigantica.

Despite the induction of high levels of systemic IgG antibodies against rSb28GST, vaccination with this recombinant $S$. bovis antigen in three different adjuvant formulations did not induce a significant level of protection against $F$. hepatica. There was no reduction in worm counts in the vaccinated animals, and no difference in the percentage of immature worms between the groups. Only slight, non-significant reductions in cumulative egg excretion were observed (up to $30 \%$ ), as compared to controls.

Calves from the FCA and Quil A adjuvant groups had significantly lower levels of $\gamma$-GT, as compared to the control group. Increased serum $\gamma$-GT levels were the only signs of pathology found after the $F$. hepatica challenge infection. Using the same infection dose as in the present experiment, Anderson et al. (1977) also reported moderately increased $\gamma$-GT levels as the only sign of parasite induced pathology. Signs of clinical fasciolosis in cattle are only to be expected after infection with 1000 cercariae (Behm and Sangster, 1999; Vercruysse and Claerebout, 2001).

The reasons why vaccination with the recombinant $S$. bovis $28-\mathrm{kDa}$ GST was unsuccessful are not clear. Immunisation with purified $F$. hepatica GST resulted in reduced worm numbers 
after homologous challenge infection in sheep (Sexton et al., 1990; Spithill et al., 1999a) and cattle (Morrison et al., 1996) and immunisation with native S. bovis GST conferred protection against homologous challenge infection in different animal species. Moreover, vaccination with the recombinant $S$. bovis $28-\mathrm{kDa}$ GST appeared to protect calves against natural $F$. gigantica infection (De Bont et al., 1997). Despite the fact that antibodies against rSb28GST cross-reacted with a $F$. hepatica $24 \mathrm{kDa}$ protein, vaccination did not result in a reduced $F$. hepatica worm burden in the present trial. Together with the observation that natural infection of sheep with $F$. gigantica induces protective immunity to homologous re-infection, while infection with $F$. hepatica does not (Spithill et al., 1997), these findings suggest that there are fundamental differences in host-parasite interactions between these two Fasciola species.

Besides a reduction in worm numbers, vaccination with schistosome 28GST also caused a decrease in worm fecundity in different Schistosoma species (Riveau and Capron, 1997). No consistent effect on worm fecundity was observed after vaccination with $F$. hepatica GST in sheep (Spithill et al., 1999a). In the present trial the only effect of vaccination on worm fecundity was a diminished embryonation rate of eggs recovered from the FCA vaccinated group. In sheep, only GST delivered in FCA induced a protective response against $F$. hepatica, while the same antigen delivered in several other adjuvants did not (Spithill et al., 1999a). In contrast, cattle were protected against $F$. hepatica by vaccination with GST in Quil A/SM, whereas GST in FCA was ineffective (Morrison et al., 1996). In the present trial the recombinant $S$. bovis $28-\mathrm{kDa}$ GST did not adequately protect cattle against F. hepatica, using either aluminium hydroxide, Quil A or FCA as adjuvant.

\section{Acknowledgements}

The authors thank N. Dierickx and I. Peelaers for their excellent technical support.

\section{References}

Anderson, P.H., Berrett, S., Brush, P.J., Herbert, C.N., Parfitt, J.W., Patterson, D.S.P., 1977. Biochemical indicators of liver injury in calves with experimental fascioliasis. Vet. Rec. 100, 43-45.

Behm, C., Sangster, N., 1999. Pathology, pathophysiology and clinical aspects. In: Dalton, J.P. (Ed.), Fasciolosis. CABI Publishing, Wallingford, UK, pp. 185-224.

Blood, D.C., Radostits, O.M. (Eds.), 1989. Veterinary Medicine. A Textbook of the Diseases of Cattle, Sheep, Pigs, Goats and Horses. Baillière Tindall, London, UK, 1502 pp.

Boulanger, D., Trottein, F., Mauny, F., Bremond, P., Couret, D., Pierce, R.J., Kadri, S., Godin, C., Sellin, E., Lecocq, J.P., Sellin, B., Capron, A., 1994. Vaccination of goats against the trematode Schistosoma bovis with a recombinant homologous schistosome-derived glutathione S-transferase. Parasite Immunol. 16, 399-406.

Capron, A., Capron, M., Riveau, G., 2002. Vaccine development against schistosomiasis from concepts to clinical trials. Br. Med. Bull. 62, 139-148.

Cervi, L., Rossi, G., Masih, D.T., 1999. Potential role for excretory-secretory forms of glutathione S-transferase (GST) in Fasciola hepatica. Parasitology 119, 627-633.

De Bont, J., 1995. Cattle schistosomiasis: host-parasite interactions. Ph.D. Thesis, Ghent University, Merelbeke, Belgium.

De Bont, J., Vercruysse, J., Grzych, J.M., Meeus, P.F.M., Capron, A., 1997. Potential of a recombinant Schistosoma bovis-derived glutathione S-transferase to protect cattle against experimental and natural S. mattheei infection. Parasitology 115, 249-255. 
Grzych, J.M., De Bont, J., Liu, J.L., Neyrinck, J.L., Fontaine, J., Vercruysse, J., Capron, A., 1998. Relationship of impairment of schistosome 28-kilodalton glutathione S-transferase (GST) activity to expression of immunity to Schistosoma mattheei in calves vaccinated with recombinant Schistosoma bovis 28-kilodalton GST. Infect. Immun. 66, 1142-1148.

Morrison, C.A., Colin, T., Sexton, J.L., Bowen, F., Wicker, J., Friedel, T., Spithill, T.W., 1996. Protection of cattle against Fasciola hepatica by vaccination with glutathione S-transferase. Vaccine 14, 1603-1612.

Mulcahy, G., Joyce, P., Dalton, J.P., 1999. Immunology of Fasciola hepatica infection. In: Dalton, J.P. (Ed.), Fasciolosis. CABI Publishing, Wallingford, UK, pp. 341-375.

Riveau, G.J., Capron, A., 1997. Vaccine strategies against schistosomiasis. In: Levine, M.M., Wooddrow, G.C., Kaper, J.B., Cobon, G.S. (Eds.), New Generation Vaccines, Second ed. Marcel Dekker, New York, NY, pp. 1081-1094.

Sexton, J.L., Milner, A.R., Panaccio, M., Waddington, J., Wijffels, G.L., Chandler, D., Thompson, C., Wilson, L., Spithill, T.W., Mitchell, G.F., Campbell, L.J., 1990. Glutathione S-transferase. Novel vaccine against Fasciola hepatica infection in sheep. J. Immunol. 145, 3905-3910.

Sirag, S.B., Christensen, N.O., Nansen, P., Monrad, J., Frandsen, F., 1981. Resistance to Fasciola hepatica in calves harbouring primary patent Schistosoma bovis infections. J. Helminthol. 55, 63-70.

Spithill, T.W., Piedrafita, D., Smooker, P.M., 1997. Immunological approaches for the control of fasciolosis. Int. J. Parasitol. 27, 1221-1235.

Spithill, T.W., Smooker, P.M., Sexton, J.L., Bozas, E., Morrison, C.A., Creaney, J., Parsons, J.C., 1999a. Development of vaccines against Fasciola hepatica. In: Dalton, J.P. (Ed.), Fasciolosis. CABI Publishing, Wallingford, UK, pp. 377-410.

Spithill, T.W., Smooker, P.M., Copeman, D.B., 1999b. Fasciola gigantica: epidemiology, control, immunology and molecular biology. In: Dalton, J.P. (Ed.), Fasciolosis. CABI Publishing, Wallingford, UK, pp. 465-525.

Trottein, F., Godin, C., Pierce, R.J., Sellin, B., Taylor, M.G., Gorillot, I., Silva, M.S., Lecocq, J.P., Capron, A., 1992. Inter-species variation of schistosome 28-kDa glutathione S-transferases. Mol. Biochem. Parasitol. 54, 63-72.

Vercruysse, J., Claerebout, E., 2001. Treatment vs. non-treatment of helminth infections in cattle: defining the threshold. Vet. Parasitol. 98, 195-214.

Vercruysse, J., Dorny, P., Hong, C., Harris, T.J., Hammet, N.C., Smith, D.G., Weatherley, A.J., 1993. Efficacy of doramectin in the prevention of gastrointestinal nematode infections in grazing cattle. Vet. Parasitol. 49, 51-59.

Yagi, A.I., Younis, S.A., Haroun, E.M., Gameel, A.A., Bushara, H.O., Taylor, M.G., 1986. Studies on heterologous resistance between Schistosoma bovis and Fasciola gigantica in Sudanese cattle. J. Helminthol. 60, 55-59. 